\title{
Intermolecular Hybridization Governs Molecular Electrical Doping
}

\author{
Ingo Salzmann, ${ }^{1, *}$ Georg Heimel, ${ }^{1, \dagger}$ Steffen Duhm, ${ }^{2}$ Martin Oehzelt, ${ }^{1,3,4}$ Patrick Pingel,,${ }^{5,1}$ Benjamin M. George,${ }^{6}$ \\ Alexander Schnegg, ${ }^{6}$ Klaus Lips, ${ }^{6}$ Ralf-Peter Blum, ${ }^{1}$ Antje Vollmer, ${ }^{4}$ and Norbert Koch $^{1,4}$ \\ ${ }^{1}$ Humboldt-Universität zu Berlin, Institut für Physik, D-12489 Berlin, Germany \\ ${ }^{2}$ Graduate School of Advanced Integration Science, Chiba University, Japan \\ ${ }^{3}$ Johannes Kepler University Linz, Institute of Experimental Physics, A-4040 Linz, Austria \\ ${ }^{4}$ Helmholtz, Zentrum Berlin für Materialien und Energie - BESSY II, D-12489 Berlin, Germany \\ ${ }^{5}$ Universität Potsdam, Institut für Physik und Astronomie, D-14476 Potsdam-Golm, Germany \\ ${ }^{6}$ Helmholtz Zentrum Berlin für Materialien und Energie - Institute for Silicon Photovoltaics, D-12489 Berlin, Germany
}

(Received 1 October 2011; published 18 January 2012)

\begin{abstract}
Current models for molecular electrical doping of organic semiconductors are found to be at odds with other well-established concepts in that field, like polaron formation. Addressing these inconsistencies for prototypical systems, we present experimental and theoretical evidence for intermolecular hybridization of organic semiconductor and dopant frontier molecular orbitals. Common doping-related observations are attributed to this phenomenon, and controlling the degree of hybridization emerges as a strategy for overcoming the present limitations in the yield of doping-induced charge carriers.
\end{abstract}

DOI: 10.1103/PhysRevLett.108.035502

Despite the pivotal role of controlled semiconductor doping for inorganic microelectronic devices, the vast majority of organic electronic devices are still based on intrinsic organic semiconductors (OSCs). While the entire field of organic electronics emerged decades ago with the discovery of metallic conduction in poly(acetylene) doped with iodine [1], using halides or alkali metals as dopants for OSCs was found to be detrimental to the processability and stability of the materials [2]. To overcome these limitations and, thus, to approach the performance of inorganic devices, global research efforts are currently directed towards applying strong organic donor or acceptor molecules as OSC dopants instead [3-5]. There, the standard approach is admixing a few mole percent of a molecular compound with an electron affinity comparable to the ionization energy (IE) of the OSC for $p$-type doping or vice versa for $n$-type doping [6]. One electron is believed to hop from the highest occupied molecular orbital (HOMO) of the OSC to the lowest unoccupied molecular orbital (LUMO) of the $p$ dopant (and vice versa for $n$-type doping), as schematically illustrated in Fig. 1(a) for the prototypical OSC pentacene (PEN) and the widely used strong electron acceptor 2,3,5,6-tetrafluoro-7,7,8,8tetracyanoquinodimethane (F4-TCNQ) as the $p$ dopant. This integer charge transfer is subsequently thought to lead to a localized electron on the $p$ dopant and an itinerant hole in the OSC matrix, resulting in an overall increase in the density of mobile charge carriers and, thus, conductivity $[3,5,7-12]$.

The most obvious implication of this mutual ionization process would be the presence of singly occupied states in the fundamental gap of the pristine OSC, that is, positive polarons $\left(\mathrm{P}^{+}\right)$, which, according to the standard model prevailing in literature $[13,14]$, exhibit a smaller IE than
PACS numbers: 81.05.Fb, 68.55.Ln, 72.80.Le, 73.61.Ph

neutral OSC molecules [Fig. 1(b)]. Upon increasing $p$-dopant concentration, the presence of these states is assumed to lead to the continuous upwards shift of the OSC HOMO towards $E_{F}$ observed in numerous ultraviolet photoelectron spectroscopy (UPS) studies [7-9,15,16]. Both this shift and the independently measured dopinginduced increase of conductivity were seen to saturate at doping levels on the order of percent $[12,16]$. Notably, the HOMO onset always remained significantly below $E_{F}$, which is in clear contrast to inorganic semiconductor physics, where the valence-band edge crosses $E_{F}$ at far lower dopant concentrations. Figure 1(c) summarizes the few (ambiguous) perceptions reported on the relative alignment of the relevant energy levels for molecularly $p$-doped OSCs in this saturated regime: Positive polarons should give rise to a density of singly occupied states directly at $[13,14]$ [solid boxes in Fig. 1(c)] or close to $E_{F}$ (dashed boxes) [8], which should appear as a photoemission feature in UPS. Most strikingly, however, such polaronic states with a reduced IE have never been observed for OSCs $p$-doped with organic acceptor molecules. Conversely, in molecularly $n$-doped OSCs, an equally saturating downwards shift of the HOMO away from $E_{F}$ was found, but, again, no (singly) occupied states at $E_{F}$ have ever been observed. Interestingly, however, new states entirely below $E_{F}$ were reported in some studies, which were designated as "gap states" but were left largely uncommented $[17,18]$.

The aim of the current study is to resolve these inconsistencies. Our principal goal was to directly observe the proposed polaron-derived states with reduced IE by UPS. Therefore, we chose to $p$-dope PEN with F4-TCNQ at an appreciable concentration of 10:1, a material pair for which efficient doping has already been reported up to 

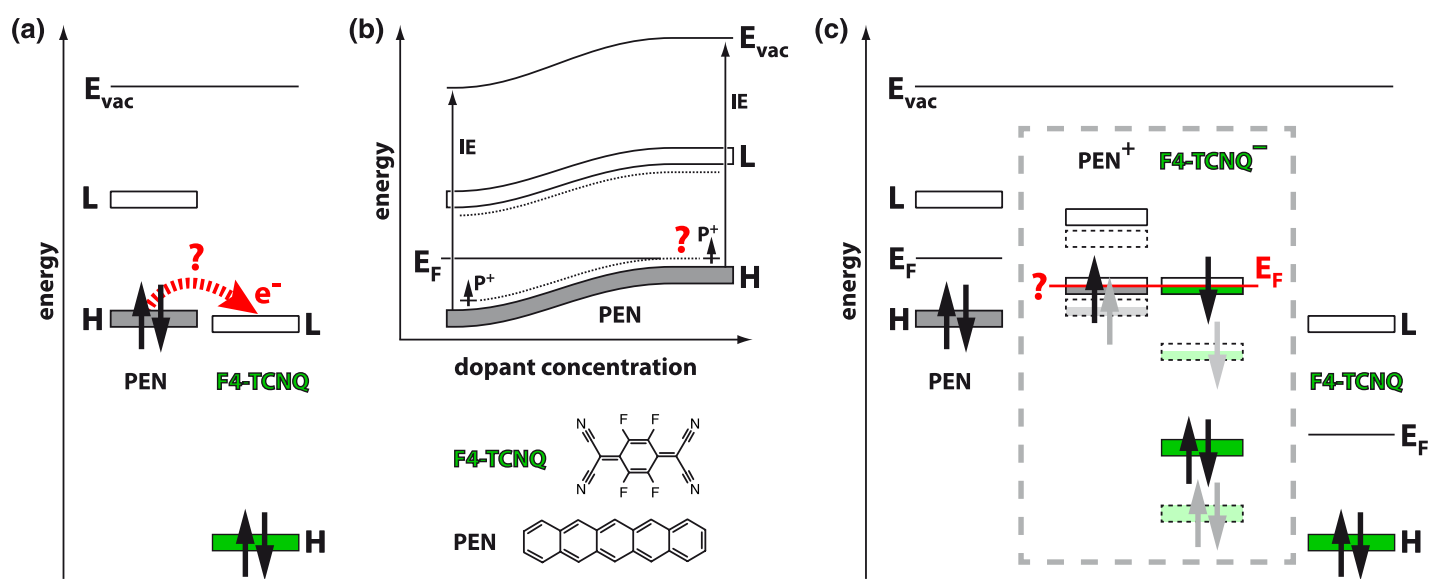

FIG. 1 (color online). (a) Schematic of the generally assumed integer electron transfer from OSC (PEN) to $p$ dopant (F4-TCNQ); $E_{\text {vac }}$ denotes the vacuum level, and $H$ and $L$ the HOMO and the LUMO levels, respectively, drawn to scale [23,24,37]. (b) Commonly observed energy-level shift upon increasing $p$-dopant concentration, which is seen to saturate at a critical doping concentration; a constant IE is retained. $H$ has never been observed to cross $E_{F}$, suggesting pinning at (singly occupied) positive polaron states $\left(\mathrm{P}^{+}\right)$. (c) Two different propositions for the energy levels in this saturation regime are shown as solid [13,14] and dashed boxes [8], respectively (center); neutral compounds are shown left and right.

that ratio [12], which is well within the saturation regime [Fig. 1(c)]. To ascertain that, if present, polaron-derived states at $E_{F}$ can indeed be experimentally observed, we further studied 1:1 mixed films [19].

The UPS spectra of these samples are shown in Fig. 2(a). A pure PEN reference film exhibits Fermi-level pinning on native silicon oxide $\left(\mathrm{SiO}_{x}\right)$ with the HOMO onset at $0.25 \mathrm{eV}$ below $E_{F}$ and an IE of $4.90 \mathrm{eV}$. This value is typical $[23,24]$ for upright standing PEN, and this molecular orientation is indeed found by the specular and grazingincidence $x$-ray diffraction results in Fig. 2(b). The 10:1 mixed PEN:F4-TCNQ film [blue curve in Fig. 2(a)] exhibits a larger IE of $5.20 \mathrm{eV}$, and no new (singly occupied) states are found at $E_{F}$ despite the appreciable doping ratio of $10 \%$. The increase of IE can be rationalized by recalling that this property depends on the molecular orientation relative to the surface [25]. As evidenced by almost vanishing diffraction features in Fig. 2(b), the film is essentially amorphous, and, therefore, an IE between the limiting values of standing $(4.9 \mathrm{eV})$ and flat-lying (5.45 eV) PEN is expected (cf. Refs. [24,26]). Increasing the mixing ratio further to 1:1 [red curve in Fig. 2(a)] still did not reveal new (singly occupied) states at $E_{F}$, but the film exhibited an entirely different electronic structure [27]. In particular, its IE increased to $5.75 \mathrm{eV}$, which is $0.85 \mathrm{eV}$ higher than that of the pure PEN film. This high IE value can no longer be attributed to orientation effects, since the highest reported solid-state IE (for flat-lying PEN) is $5.45 \mathrm{eV}$ [24], and, furthermore, the completely vanished diffraction features in Fig. 2(b) prove our film to be amorphous [28].

Underlining the generality of these observations, almost identical results were obtained on a different substrate, the conducting polymer poly(ethylenedioxythiophene):poly (styrenesulfonate), where an IE increase of $0.75 \mathrm{eV}$ was observed between pristine PEN and the 1:1 mixed film. Likewise, a different OSC, $\alpha$-sexithiophene, 1:1 mixed with F4-TCNQ on $\mathrm{SiO}_{x}$ led to qualitatively identical results with an IE increase of $0.65 \mathrm{eV}$ [27].
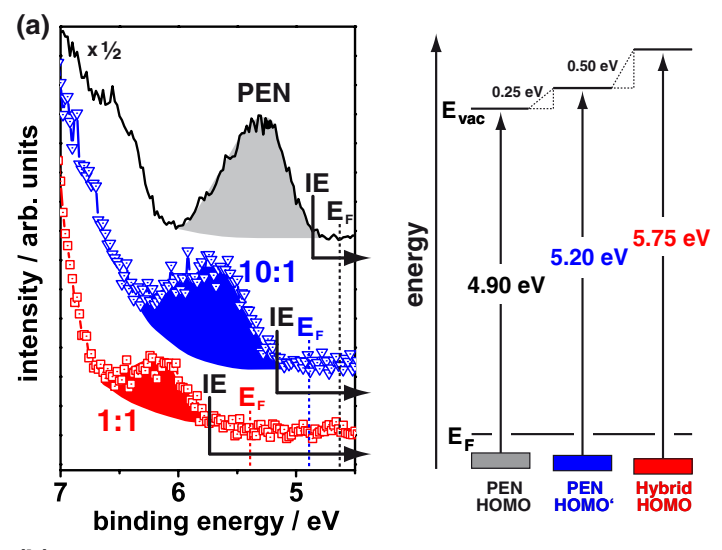

(b)
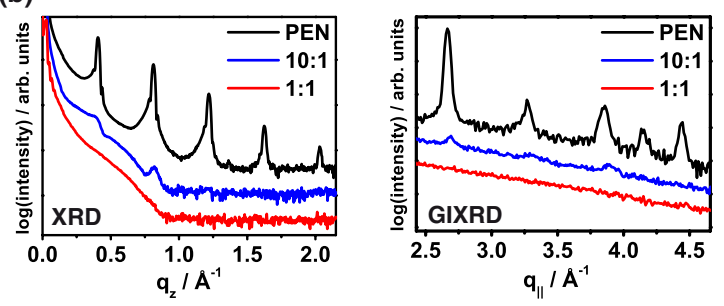

FIG. 2 (color online). (a) Left: Synchrotron UPS results $(h \nu=$ $35 \mathrm{eV}$ ) in the range of $E_{F}$ for $20 \mathrm{~nm} \mathrm{PEN} \mathrm{on} \mathrm{SiO}_{x}$ (black curve), 10:1 doped (blue curve), and 1:1 mixed (red curve) PEN:F4TCNQ films; binding energy is given with respect to $E_{\mathrm{vac}}$. Right: Corresponding ionization energies. (b) Synchrotron specular and grazing-incidence x-ray diffraction results; $\mathbf{q}_{z}$ and $\mathbf{q}_{\|}$denote the scattering vector components perpendicular and parallel to the substrate, respectively. All spectra are vertically offset for the sake of clarity. 
Our experimental findings clearly disagree with the prevailing model of OSC doping (Fig. 1), which fails to capture both the observed IE increase and the absence of photoemission intensity at $E_{F}$. To fully explain our results and the fundamental mechanism of molecular electrical $p$ doping in general, we suggest intermolecular orbital hybridization between the OSC HOMO and the dopant LUMO, leading to the formation of a doubly occupied bonding and an empty antibonding supramolecular hybrid orbital [Fig. 3(a)]. To substantiate this hypothesis, we performed density-functional theory (DFT) calculations. As the mixed films are amorphous and, therefore, the relative orientation of PEN and F4-TCNQ in the solid state is inaccessible, we considered isolated molecules and OSC-dopant complexes instead. However, we chose a hybrid DFT functional that reproduces the experimental HOMO and LUMO energies of PEN and F4-TCNQ in the solid state, thus mimicking the impact of polarization effects [Fig. 3(a)]; isosurface plots of the relevant frontier molecular orbitals are shown in Fig. 3(b). From a total of six initial guesses, where the molecules were rotated and/or laterally shifted with respect to each other to assure a reasonable sampling of configuration space, only three nonsymmetric local-minimum structures emerged where maximized orbital overlap leads to a substantial splitting of bonding and antibonding hybrid orbitals; indications for such a splitting have also been reported in Refs. [29-31] for related systems. Figure 3(b) displays one representative configuration, where the molecules are azimuthally rotated by $19^{\circ}$, and the corresponding supramolecular hybrid orbitals are shown in Fig. 3(a). The calculated intermolecular binding energies are substantial and essentially identical for all three configurations $(0.87-0.94 \mathrm{eV})$. This correlates well with our experimental finding of amorphous films and presents an appreciable driving force for the preferential formation of such complexes. Notably, their calculated fundamental gaps are $1.07-1.43 \mathrm{eV}$, which is at least $1.11 \mathrm{eV}$ lower than that computed for pristine PEN (2.54 eV) [27].

The most important consequence of this model is that, upon doping with F4-TCNQ, both electrons from the PEN HOMO occupy the new (bonding) hybrid orbital, rather than one electron hopping from the PEN HOMO to the F4-TCNQ LUMO [cf. Fig. 1(a)]. Our calculations not only support that the hybridized PEN-F4-TCNQ complexes have a higher IE than pristine PEN, which is fully consistent with our UPS results [Fig. 2(a)], but also indicate that they exhibit a reduced energy gap [Fig. 3(a)]. The latter finding was experimentally validated by ultraviolet-visible (UV-VIS) spectroscopy [Fig. 3(c)]. The pure PEN and F4-TCNQ reference films show fundamental absorption maxima at 1.85 and $2.85 \mathrm{eV}$, respectively. The 10:1 doped film indeed exhibits features at lower energies $(1.28$ and $1.42 \mathrm{eV})$, which increase in the $1: 1$ mixed film. To assess the concentration of charged molecules (polarons) in the films, we performed continuous wave electron paramagnetic resonance (cwEPR) experiments on the 10:1 and 1:1 mixed films as well as on pristine PEN powder for a reference [Fig. 3(d)] [32]. Given that the cwEPR signal and, hence, the polaron density decreases with increasing

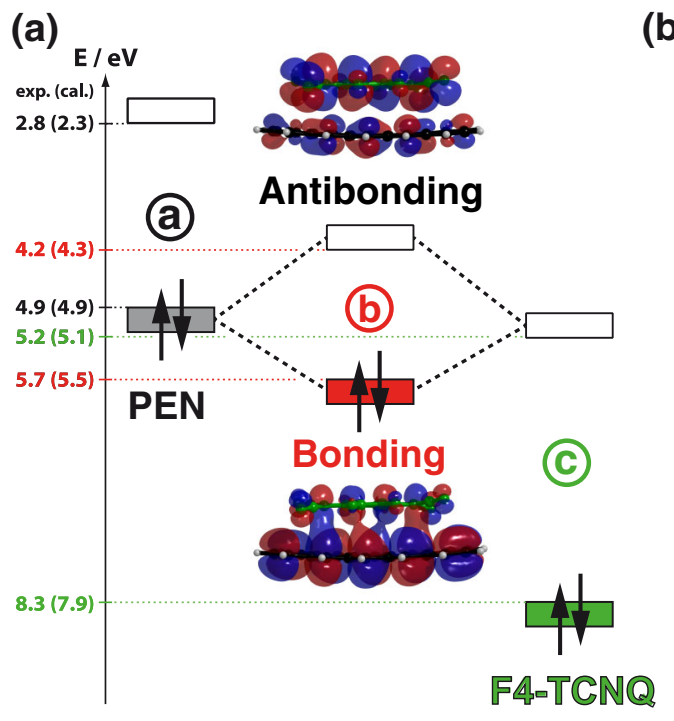

(b)
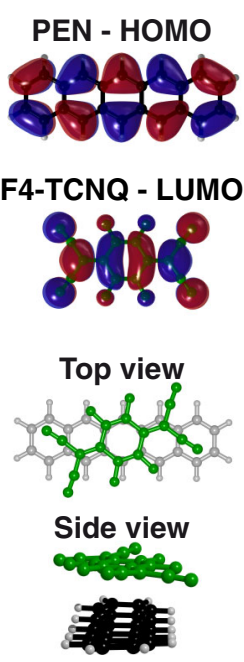

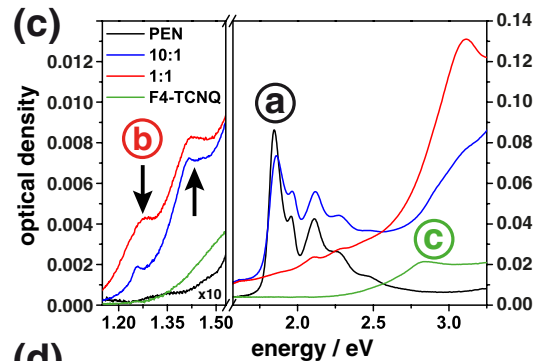

(d)

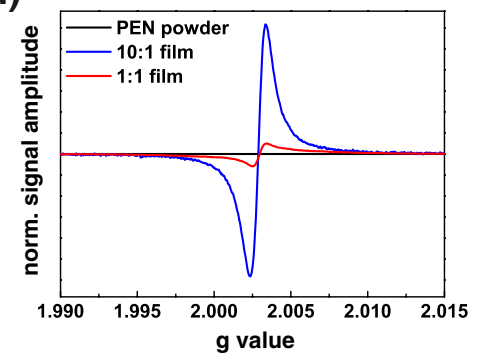

FIG. 3 (color online). (a) Schematic of frontier-orbital hybridization and orbital isosurface plots for a PEN-F4-TCNQ complex. Circled a, b, and c indicate optical transitions assigned in (c); experimental energy values (exp.) taken from Fig. 2(a), Ref. [7] (F4TCNQ), and Ref. [37] (PEN LUMO), and that for the empty antibonding hybrid orbital estimated via the optical gap assuming an exciton binding energy equal to that of PEN; DFT values (cal.) given in parentheses. (b) Top: Individual PEN HOMO and F4-TCNQ LUMO orbitals; bottom: representative local-minimum mutual orientation. (c) Experimental UV-VIS absorption spectra; transitions assigned to pristine PEN (circled a), the PEN-F4-TCNQ complex (circled b) in films of equal PEN content and to pristine F4-TCNQ (circled c) for a drop-cast reference film. (d) Corresponding cwEPR spectra normalized to number of molecules in the respective films. 
doping ratio, the UV-VIS absorption features are attributed to transitions from the bonding to the antibonding supramolecular hybrid orbitals [Fig. 3(a)] [33].

Our model of frontier-orbital hybridization can successfully explain common doping-related observations, as illustrated in Fig. 4(a) for $p$-type doping. (i) In the general case, where the OSC HOMO is not Fermi-level pinned, $E_{F}$ lies well within the gap of the pristine material (left panel). Introducing the molecular acceptor results in hybrid states, low-lying unoccupied states in the fundamental gap of the OSC, in particular (center panel). In contrast to inorganic semiconductors, where acceptor levels are typically only a few $\mathrm{meV}$ above the valence-band edge, the empty hybrid states are still several tenths of an $\mathrm{eV}$ above the HOMO of the pristine OSC [cf. Fig. 3(a)]. As all available states are occupied following Fermi-Dirac statistics, only a fraction of the hybridized OSC-dopant complexes are ionized at room temperature. This rationalizes the comparably high doping concentrations (on the order of mole percent) needed in practical applications [4], which conflicted with the notion of every dopant providing one charge carrier [cf. Fig. 1(a)]. (ii) As a further consequence of Fermi-Dirac occupation, the OSC HOMO gradually shifts toward $E_{F}$ upon increasing doping ratio [cf. Fig. 1(c)]. Importantly, this shift saturates when $E_{F}$ is stabilized between the pristine OSC HOMO and the unoccupied hybrid states [center panel in Fig. 4(a)]. The thusly reduced barrier for hole injection from the substrate $E_{F}$ to the OSC $\mathrm{HOMO}$, the increased density of charge carriers, and, at low doping ratios, an initial increase in their mobility through prior trap filling by the first generated charges $[5,21,34]$ all contribute to a marked decrease in device resistance [3,5,7-12]. (iii) No new (positive polaron) states
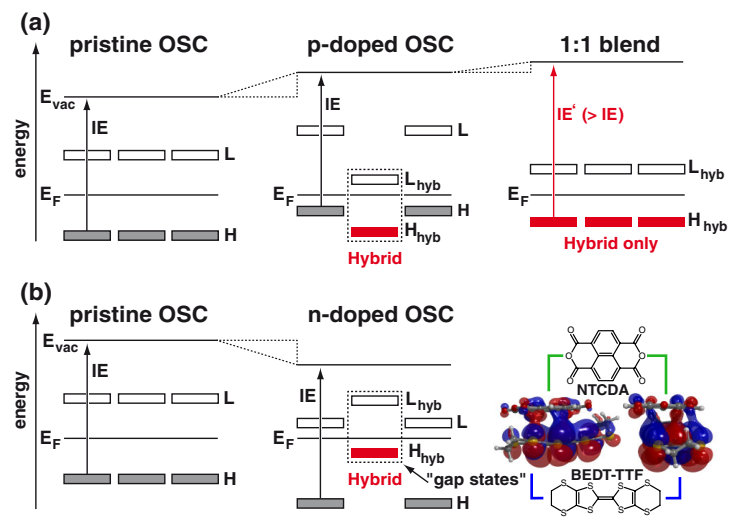

FIG. 4 (color online). (a) Schematic energy-level diagram for molecular electrical $p$ doping via OSC-dopant frontier-orbital hybridization. $H_{\text {hyb }}$ and $L_{\text {hyb }}$ denote the bonding and antibonding supramolecular hybrid orbital, respectively, and $\mathrm{IE}^{\prime}$ the increased hybrid IE observed here in the 1:1 blend of OSC and dopant. (b) $n$-type doping proceeds in full analogy to (a); right: chemical structures and calculated bonding hybrid orbitals for the prototypical material pair 1,4,5,8-naphthalene-tetracaboxylicdianhydride and bis(ethylenedithio)-tetrathiafulvalene. can be observed at $E_{F}$, because the doubly occupied bonding hybrid states must always be lower in energy than the OSC HOMO (center panel). At typical doping ratios of a few percent, the UPS signature arising from the occupied density of states of the pristine OSC thus masks that of the bonding supramolecular hybrid orbitals (cf. Ref. [8]). As shown in the present study for PEN and F4-TCNQ, these orbitals and their increased $\mathrm{IE}^{\prime}$ can be observed upon 1:1 mixing of OSC and dopant (right panel). Calculations on further material pairs $[7,8,35]$ underline that hybridization of OSC and dopant frontier molecular orbitals is likely a general phenomenon [27].

$n$-type doping proceeds in full analogy [Fig. 4(b)]: The OSC LUMO hybridizes with the HOMO of the dopant, which leads to doubly occupied bonding hybrid states in the OSC gap. Consequently, the OSC LUMO shifts toward $E_{F}$ upon increasing dopant concentration. This shift saturates when the Fermi level is stabilized between the OSC LUMO and the occupied hybrid orbitals, which account for the gap states observable by UPS on molecularly $n$-doped OSCs $[17,18]$. A marked decrease of device resistance then follows from the corresponding factors outlined for $p$-type doping above. As an example for hybridization between OSC and $n$ dopant, the calculated bonding hybrid orbital of the prototypical material pair 1,4,5,8-naphthalene-tetracaboxylic-dianhydride and bis (ethylenedithio)-tetrathiafulvalene $[3,36]$ is shown in Fig. 4(b).

In summary, we presented experimental and theoretical evidence for intermolecular hybridization between the frontier orbitals of OSC and molecular dopant. In contrast to the integer charge-transfer model prevalent in literature, the presence of supramolecular hybrid orbitals in the fundamental gap of the pristine OSC (empty for $p$-type and occupied for $n$-type doping) provides a natural explanation for commonly observed phenomena in molecularly doped OSCs. Even more importantly, our model reveals the limitations of current molecular dopants: Because of the substantial offset between OSC frontier orbital and intragap hybrid states, only a fraction of the OSC-dopant complexes are ionized at finite temperature, thus limiting the achievable maximum density of mobile charge carriers in the OSC and, hence, its conductivity. To reach higher carrier densities without adversely affecting their mobility through deterioration of the OSC solid-state packing [cf. Fig. 2(b)], that is, at lower doping concentrations than presently used, this offset must be minimized by suppressing the splitting between bonding and antibonding hybrid orbitals. Since the splitting is due to electronic coupling between OSC and dopant frontier molecular orbitals, the coupling must be inhibited. Shielding the functional core of sufficiently strong acceptor and donor molecules through the attachment of bulky side groups thus emerges as a design strategy for improved molecular dopants in future high-performance organic electronic devices. 
We thank D. Neher (Universität Potsdam) for UV-VIS measurements, A. Elschner (H. C. Starck) for providing poly(ethylenedioxythiophene):poly(styrenesulfonate), W. Caliebe (DESY, HASYLAB) for experimental support, and J.P. Rabe (HU-Berlin) for fruitful discussions. Financing through the DFG (Germany), the JSPS (Japan), and Austrian Science Fund (FWF) Project No. P21094 is gratefully acknowledged.

*ingo.salzmann@physik.hu-berlin.de †georg.heimel@physik.hu-berlin.de

[1] H. Shirakawa, E. J. Louis, A. G. MacDiarmid, C. K. Chiang, and A.J. Heeger, J. Chem. Soc. Chem. Commun. 1977, 578 (1977).

[2] G. Parthasarathy, C. Shen, A. Kahn, and S. R. Forrest, J. Appl. Phys. 89, 4986 (2001).

[3] M. Pfeiffer, T. Fritz, J. Blochwitz, A. Nollau, B. Plnnigs, A. Beyer, and K. Leo, Controlled Doping of Molecular Organic Layers: Physics and Device Prospects, Advances in Solid State Physics Vol. 39 (Springer, Berlin, Heidelberg, 1999), pp. 77-90.

[4] S. Reineke, F. Lindner, G. Schwartz, N. Seidler, K. Walzer, B. Lussem, and K. Leo, Nature (London) 459 , 234 (2009).

[5] K. Walzer, B. Maennig, M. Pfeiffer, and K. Leo, Chem. Rev. 107, 1233 (2007).

[6] K. Harada, A. G. Werner, M. Pfeiffer, C. J. Bloom, C. M. Elliott, and K. Leo, Phys. Rev. Lett. 94, 036601 (2005).

[7] W. Y. Gao and A. Kahn, J. Phys. Condens. Matter 15, S2757 (2003)

[8] W. Y. Gao and A. Kahn, J. Appl. Phys. 94, 359 (2003).

[9] W. Y. Gao and A. Kahn, Appl. Phys. Lett. 79, 4040 (2001).

[10] S. D. Ha and A. Kahn, Phys. Rev. B 80, 195410 (2009).

[11] K. Harada, M. Riede, K. Leo, O. R. Hild, and C. M. Elliott, Phys. Rev. B 77, 195212 (2008).

[12] K. Harada, M. Sumino, C. Adachi, S. Tanaka, and K. Miyazaki, Appl. Phys. Lett. 96, 253304 (2010).

[13] M. Lögdlund, R. Lazzaroni, S. Stafström, W. R. Salaneck, and J. L. Bredas, Phys. Rev. Lett. 63, 1841 (1989).

[14] D. Steinmüller, M. G. Ramsey, and F. P. Netzer, Phys. Rev. B 47, 13323 (1993).

[15] A. Kahn, W. Zhao, W. Y. Gao, H. Vazquez, and F. Flores, Chem. Phys. 325, 129 (2006).

[16] S. Olthof, W. Tress, R. Meerheim, B. Lüssem, and K. Leo, J. Appl. Phys. 106, 103711 (2009).

[17] C. K. Chan, W. Zhao, S. Barlow, S. Marder, and A. Kahn, Org. Electron. 9, 575 (2008).

[18] C. Falkenberg, C. Uhrich, S. Olthof, B. Maennig, M. K. Riede, and K. Leo, J. Appl. Phys. 104, 034506 (2008).

[19] Direct interaction between F4-TCNQ and supporting substrates can lead to the HOMO of a subsequently deposited OSC shifting towards $E_{F}$ [20]. As F4-TCNQ can diffuse through OSCs [8,9,21,22], this interfacial effect might be misinterpreted as being related to PEN-F4-TCNQ interaction in the bulk. To minimize its potential impact, we chose $\mathrm{SiO}_{x}$ with a work function in the range of the PEN IE as the substrate, leading to Fermi-level pinning of the PEN HOMO [23]; i.e., the HOMO is essentially stabilized at a minimal energetic distance from $E_{F}$, and potential diffusion of F4-TCNQ to the substrate-OSC interface cannot affect our results; for UPS data of 1:1 mixed films on F4-TCNQ precovered substrates, see [27].

[20] N. Koch, S. Duhm, J.P. Rabe, A. Vollmer, and R. L. Johnson, Phys. Rev. Lett. 95, 237601 (2005).

[21] F. Ali, N. Periasamy, M.P. Patankar, and K. L. Narasimhan, J. Appl. Phys. 110, 044507 (2011).

[22] S. Duhm, I. Salzmann, B. Broker, H. Glowatzki, R. L. Johnson, and N. Koch, Appl. Phys. Lett. 95, 093305 (2009).

[23] I. Salzmann, S. Duhm, G. Heimel, M. Oehzelt, R. Kniprath, R. L. Johnson, J. P. Rabe, and N. Koch, J. Am. Chem. Soc. 130, 12870 (2008).

[24] H. Fukagawa, H. Yamane, T. Kataoka, S. Kera, M. Nakamura, K. Kudo, and N. Ueno, Phys. Rev. B 73, 245310 (2006).

[25] S. Duhm, G. Heimel, I. Salzmann, H. Glowatzki, R. L. Johnson, A. Vollmer, J.P. Rabe, and N. Koch, Nature Mater. 7, 326 (2008).

[26] N. Sato, K. Seki, H. Inokuchi, and Y. Harada, Chem. Phys. 109, 157 (1986).

[27] See Supplemental Material at http://link.aps.org/ supplemental/10.1103/PhysRevLett.108.035502 for UPS and $\mathrm{X}$-ray photoemission spectroscopy data, conductivity data, further DFT results and benchmark calculations, materials, and methods.

[28] This is an important finding by itself, as it experimentally confirms the doping-induced deterioration of the OSC solid-state packing held responsible for the observed reduction of charge-carrier mobility at high doping ratios and, consequently, the saturation of conductivity $[12,16]$.

[29] K. F. Braun and S. W. Hla, J. Chem. Phys. 129, 064707 (2008).

[30] F. Jäckel, U. G. E. Perera, V. Iancu, K. F. Braun, N. Koch, J. P. Rabe, and S. W. Hla, Phys. Rev. Lett. 100, 126102 (2008).

[31] E. E. Aziz, A. Vollmer, S. Eisebitt, W. Eberhardt, P. Pingel, D. Neher, and N. Koch, Adv. Mater. 19, 3257 (2007).

[32] V. V. N. Ravi Kishore, N. Periasamy, B. Bhattacharjee, R. Das, P. L. Paulose, and K. L. Narasimhan, Chem. Phys. Lett. 367, 657 (2003).

[33] The presence of two peaks instead of one can be rationalized either by a vibronic fine structure of the fundamental hybrid transition or by two dominant mutual molecular orientations with slightly different optical gaps.

[34] D. Ray and K. L. Narasimhan, J. Appl. Phys. 103, 093711 (2008).

[35] S. P. Tiwari, W. J. Potscavage, T. Sajoto, S. Barlow, S. R. Marder, and B. Kippelen, Org. Electron. 11, 860 (2010).

[36] A. Nollau, M. Pfeiffer, T. Fritz, and K. Leo, J. Appl. Phys. 87, 4340 (2000).

[37] A. Kahn, N. Koch, and W. Y. Gao, J. Polym. Sci., Part B: Polym. Phys. 41, 2529 (2003). 